\title{
Surgical resection of primary tumor site is associated with prolonged survival in metastatic non-functioning pancreatic neuroendocrine tumors
}

\author{
Xavier M. Keutgen, MD1, Naris Nilubol, MD¹, Joanne Glanville, MD¹, Samira M. Sadowski, \\ MD1', David J. Liewehr, MS², David J. Venzon, PhD $^{2}$, Seth M. Steinberg, PhD², and Electron \\ Kebebew, MD ${ }^{1}$
}

${ }^{1}$ Endocrine Oncology Branch, Center for Cancer Research, National Cancer Institute, National Institutes of Health, Bethesda, Maryland ${ }^{2}$ Biostatistics and Data Management Section, Office of the Clinical Director, Center for Cancer Research, National Cancer Institute, National Institutes of Health, Bethesda, Maryland

\section{Abstract}

Background-Non-functioning pancreatic neuroendocrine tumors (NFpNET) present with distant metastases in up to $50 \%$ of patients. It is unknown whether removal of the primary tumor in patients with NFpNET and metastases is beneficial.

Methods-We used the Surveillance, Epidemiology and End Results (SEER) database to identify patients with NFpNET and distant metastases. The primary outcome measure in this study was overall survival.

Results-We identified 882 patients with metastatic NFpNET and survival data. 303 (34\%) patients had surgical removal of their primary tumor of which $243(80 \%)$ were grade I or II. Median survival of patients undergoing surgery was 65 (95\% CI: 60-86) versus 10 (8-12) months for those without surgery $(\mathrm{p}<0.0001)$. Patients diagnosed after $2003(\mathrm{n}=625,71 \%)$ were more likely to undergo an operation than those diagnosed earlier $(\mathrm{p}=0.001)$. Multivariable analysis showed that lower tumor grade $(\mathrm{p}<0.0001)$, younger age $(\mathrm{p}<0.0001)$, diagnosis during or after 2003 ( $\mathrm{p}=0.0003$ ), tumor site in the body/tail $(\mathrm{p}=0.009)$ and surgical resection of the primary tumor site $(p<0.0001)$ were significantly associated with prolonged survival of patients with NFpNET and distant metastases.

Conclusions-This study suggests that surgical removal of primary NFpNET is associated with longer survival in patients with distant metastases and could therefore be considered as a additional treatment option in this patient population.

Correspondence: Electron Kebebew, kebebewe@ mail.nih.gov, Tel: 301-496-5049, Address: Endocrine Oncology Branch (EOB), National Cancer Institute (NIH), 10 Center Drive, 3W-5840, Bethesda, Maryland, 20892, USA.

Publisher's Disclaimer: This is a PDF file of an unedited manuscript that has been accepted for publication. As a service to our customers we are providing this early version of the manuscript. The manuscript will undergo copyediting, typesetting, and review of the resulting proof before it is published in its final citable form. Please note that during the production process errors may be discovered which could affect the content, and all legal disclaimers that apply to the journal pertain. 


\section{INTRODUCTION}

Pancreatic neuroendocrine tumors (pNET) are a rare form of pancreatic neoplasm. The incidence however is continuously increasing in the US with an estimate of 3-5 new cases per million inhabitants per year (1-3). pNET can be divided into functioning tumors that secrete hormones such as insulin, glucagon, gastrin, vasoactive intestinal peptide (VIP) and somatostatin, and non-functioning tumors (NFpNET), that lack clinical symptoms despite actively secreting peptides such as chromogranin A and pancreatic polypeptide $(4,5)$. NFpNET are more common than functional tumors and are often diagnosed in a more advanced stage, presenting with distant metastases in $60 \%$ of cases (2). This may be due to a delay in diagnosis because of the lack of overt clinical symptoms when compared to functional tumors (4). Most NFpNET are sporadic; however, they can occur as part of familial cancer syndromes such as Von Hippel-Lindau Syndrome (VHL), Multiple Endocrine Neoplasia Type 1 (MEN1) or tuberous sclerosis. NFpNET due to genetic disorders (mutations in $V H L, M E N 1, T S C 1 / 2)$ usually have an indolent natural history and are often managed non-operatively when compared to their sporadic counterparts $(5,6)$. Previous studies have identified important prognostic factors for pNET, including tumor grade (4). In 2010, the World Health Organization (WHO) classification defined 3 classes of grades: Grade 1 tumors, with $<2$ mitosis/10 HPF and Ki-67 index $<2 \%$; Grade 2 tumors, with 2-20 mitosis/10 HPF and Ki-67 index 3-20\% and Grade 3 tumors, with >20 mitosis/10 HPF and Ki-67 index of $>20 \%(7,8)$. The current American Joint Committee on Cancer (AJCC) staging of pNET includes tumor size, lymph node and distant metastases. However, it remains controversial whether tumor size and presence of lymph node metastases truly are associated with worse prognosis $(2,9)$. Tumor grading has not been included in the AJCC staging as of yet (9). The presence of distant metastases may represent the most important prognostic factor. Surgical intervention remains the gold standard for patients with well or moderately differentiated pNET and advanced stage, if $>90 \%$ of tumor burden can be removed (7). It is unclear however, if patients with NFpNET and distant metastases benefit from removal of their primary tumor. Both the European Neuroendocrine Tumor Association (ENETS) and North American Neuroendocrine Tumor Association (NANETS) guidelines currently do not make any recommendations on whether the primary tumor should be removed in this clinical scenario $(4,7)$. The aim of this study was to evaluate the impact of surgical resection of the primary tumor in patients with NFpNET and distant metastases, as well as indentify variables associated with prolonged survival in this patient population.

\section{METHODS}

The primary endpoint of this study was to identify factors associated with prolonged overall survival in a large patient cohort of NFpNET and distant metastases. The secondary endpoints were to determine additional prognostic factors associated with overall survival when stratified by surgery, and to determine which factors may be associated with the likelihood of patients undergoing surgery. 


\section{Data acquisition}

The Surveillance, Epidemiology and End Results (SEER) program of the National Cancer Institute is a US population-based cancer registry that collects information on patient demographics, epidemiology, tumor characteristics, initial treatment, tumor histopathology and stage at diagnosis, and follow-up vital status since 1973. The Office of Human Subject Research at the National Institutes of Health exempted our study from approval, since the SEER data contains unidentifiable patient information. Cases and detailed information were captured using SEER*Stat software (Version 8.1.5 - March 31, 2014; Cancer Statistics Branch, NCI, Bethesda, MD).

We identified patients diagnosed with NFpNET from 1973 to 2011. The histologic subtypes and their International Classification of Disease codes (ICD-O-3) included in the cohort were: large cell neuroendocrine carcinoma (8013), islet cell carcinoma (8150) and neuroendocrine carcinoma (8246) (10). Cases with codes for mixed or functioning pNET were excluded. Only patients with distant metastases according to the "SEER historic stage" variable or American Joint Commission on Cancer (AJCC) stage IV patients were included in this dataset. The variables included in the analyses were histologic differentiation grade (well-differentiated: defined as grade I, moderately differentiated: defined as grade II, and poorly differentiated/undifferentiated: defined as grade III), age, sex, race/ethnicity (white, black, other), tumor location (head, body, tail, overlapping), year of diagnosis, lymph node metastases, site specific surgery (enucleation, local resection, partial pancreatectomy, total pancreatectomy, pancreatectomy-not otherwise specified), resection of primary and surgical procedure of other sites as well as survival time and cause-specific death (11). Because TNM staging system has been specifically coded in a separate category since 2004, tumor size was reclassified into T-Stage per the AJCC staging system (12).

\section{Statistical Analysis}

A set of actuarial analyses on survival data beginning at date of diagnosis as reported in the SEER database using the Kaplan-Meier method was performed. The difference in survival among groups of patients was determined using a log-rank test. Survival times were censored if the patient was alive at last follow-up. Median survival times and associated 95\% confidence intervals were calculated; if the upper limit of the confidence interval could not be calculated because it would extend beyond the range of the data, it was labeled as undefined. After eliminating variables for which there was no evidence that they were associated with overall survival, or combining strata that were similar, a multivariable Cox proportional hazards regression analysis on the remaining variables was performed (surgery, year of diagnosis, grade, age, tumor location). Both stepwise and backward selection methods were used. Among the variables remaining in the model, two-way interaction effects were tested. Multivariable logistic regression analysis was used to determine the simultaneous impact of factors, which were associated with performing surgery or not. The association between surgery or not and dichotomous parameters was determined by Fisher's exact test, while the association with ordered categorical parameters was determined by a Cochran-Armitage test. All p-values were two-tailed, not adjusted for multiple comparisons, and considered as statistically significant if $\mathrm{p}<0.05$. 


\section{RESULTS}

Of 882 patients with histologically confirmed NFpNET and distant metastases, 34\% (303/882) underwent surgical resection of their primary tumor. Twenty nine percent (83/289) had additional resection of distant metastases. Sixty-six percent (579/882) of patients did not undergo surgery. The histologic differentiation grade was I in 39\%, II in $22 \%$ and III in $39 \%$ of patients ( $\mathrm{n}=882$ ). Compared to patients who did not undergo surgery, patients in the surgery group were significantly younger ( $<65$ age) ( $75 \%$ vs. $53 \%$, $p<0.0001$ ), had a greater percentage of grade I tumors (54\% vs. $31 \%$ ), similar percentage of grade II tumors ( $26 \%$ vs. $20 \%$ ), but lower percentage of grade III tumors ( $20 \%$ vs. $49 \%$, $p<0.0001$ for overall trend), a higher percentage of tumors located in the body/tail of the pancreas $(54 \%$ vs. $29 \%, p<0.0001)$ and a higher rate of lymph node metastases $(71 \%$ vs. $39 \%, p<0.0001$ ), which may be due to lymph node sampling during resection (Table 1).

Median survival of patients undergoing surgery was significantly longer than those without surgery (5.42 years (95\% CI: 5.00 to 7.17 years) vs. 0.83 years (95\% CI: 0.67 to 1.00 years) $\mathrm{p}<0.0001$ ). Median survival by individual clinical or tumor characteristics is summarized in Table 2. A comparison of estimated overall survival by clinical or tumor characteristics is shown in Figure 1. There was a trend towards significantly longer overall survival when primary tumor and distant metastatic sites were removed $(\mathrm{n}=83)$ compared to that of patients who had only primary tumors removed alone $(n=206)(8.50$ years $(95 \%$ CI: 8.42 years to undefined vs. 5.58 years ( $95 \%$ CI: 4.00 to 6.58 years), $\mathrm{p}=0.052$ ). 18 patients underwent removal of distant metastases only; their median survival time was 5.33 years (95\% CI: 0.67 to undefined). The data for these 18 patients were not included in the primary analysis of 303 patients. Furthermore, among patients with grade III tumors $(n=341)$, removal of the primary tumor was associated with longer survival when compared to those patients without surgical intervention $(1.25$ years $(0.75-2.17 ; \mathrm{n}=60)$ versus 0.25 years $(0.17-0.42 ; \mathrm{n}=281)$, $\mathrm{p}<0.0001$, HR $0.42(0.30-0.59))$.

Univariate analysis yielded that lower tumor grade (I/II vs. III), age ( $<65$ vs. $\ 65$ ), year of diagnosis (<2003 vs. 2003), tumor location (head/overlapping vs. body/tail), lymph node metastases (yes vs. no) and surgical resection (yes vs. no) were associated with prolonged survival, but not race/ethnicity, sex, tumor size or site specific surgery (Table 3). Of note, there was a highly significant association ( $\mathrm{p}<0.0001$, data not shown) between surgery and lymph node metastases. Within the surgery group, survival was not significantly different according to lymph node positivity. Independent prognostic factors associated with overall mortality on multivariable analysis included low grade $(\mathrm{I} / \mathrm{II})$, young age $(<65)$, year of diagnosis $\geq 2003$, primary tumor location in the body/tail of the pancreas and surgical removal of primary site (Table 4). However, while this model demonstrates the effect of surgery after accounting for the simultaneous effect of several other parameters, one curve in Figure 1D has a hazard that changes slowly over time while the other curve has a much higher hazard in the earlier years than in the later. This difference in hazards may limit the validity of the actual parameter estimates presented. 


\section{Subgroup Analysis}

When analyses are performed separately in the groups receiving surgery or not for the primary tumor, the analyses better illustrate the magnitude of the effects of the other parameters for the two groups of patients, including some important differences between the two groups. When performing a multivariable analysis on all 303 patients who underwent removal of their primary tumor, only year of diagnosis after 2003 and low tumor grade (I/II) were simultaneously associated with prolonged survival in our patient population. When analyzing all variables of the 579 patients who did not undergo surgery, lower tumor grade, more recent year of diagnosis and younger age were simultaneously associated with prolonged survival. Tumor location in the body or tail of the pancreas was only marginally associated with prolonged survival in these patients (Table 5).

\section{Factors associated with patients undergoing surgery}

To gain insight on patient selection, we analyzed the factors associated with removal of the primary tumor from patients with NFpNETs and distant metastases, using a logistic regression model stratified by year of diagnosis since that was not a controllable factor. We found that, well or moderately differentiated tumors (grade I/II) and tumor location in the body/tail of the pancreas were significantly associated with removal of primary tumor, independently of the time period in which patients were diagnosed. In the time period after 2003 , age $<65$ was also associated with removal of primary tumor (Table 6). Of interest, it was also determined that among the patients diagnosed at or after 2003, there was evidence for an interaction between age and tumor location $(\mathrm{p}=0.011)$. Specifically, older patients ( 65 ) with tumors located in the head/other of the pancreas were much less likely to have surgery than other patients, but especially compared to younger patients with tumors in the body/tail of the pancreas.

\section{DISCUSSION}

Surgery remains the only curative treatment for functioning and non-functioning pNET. Surgical resection is usually performed when all tumor can be completely resected or if debulking of more than $90 \%$ of tumor burden can be achieved (7). It remains unknown however, if surgical resection of the primary tumor is beneficial in the setting of distant metastases. Both ENETS and NANETS guidelines currently do not recommend routine surgical resection in patients with NFpNET and distant metastasis since the available data supporting this strategy are sparse $(4,7)$.

Our study, using data from 882 patients from the SEER database, is the largest report to date analyzing the effects of primary tumor resection in NFpNET with distant metastases. Our findings show that resection of primary tumor is independently associated with longer survival in these patients on multivariable analysis. Additionally our results shed light on other factors associated with prolonged survival, such as younger age, well- or moderately differentiated tumor grade as well as tumor location and more recent year of diagnosis. These variables may be important when considering surgical intervention in patients with NFpNET and distant metastases. A previous study examining biological behavior and outcome of 2158 NFpNET using the SEER database included 614 patients with distant 
metastases and also demonstrated that median survival time of patients undergoing surgical resection of primary tumor was significantly longer than patients who did not undergo resection (3.5 vs. 1.0 years, $\mathrm{p}<0.001$ ) (2). Although these results are similar to our findings, the survival time was longer in our analysis and we identified additional variables associated with prolonged survival in this patient population. In contrast, a single-institution study looking at 51 patients with NFpNET and distant metastases, found that there was no statistically significant difference in median survival when comparing 19 patients undergoing resection of primary tumors (54.3 months, CI 25-83.6) to 32 patients without resection (39.5 months, CI 5.4-73.6). This finding was true even when considering only well- and moderately differentiated (Ki-67 $<10 \%)$ tumors, despite a prolonged median survival of 69.3 months (CI 45.9-88.7) in the surgery group versus 54.8 months (CI 0.0149.1 ) in the non-surgery group (13). Despite a longer survival time in the surgery group, the results of this study may not have been statistically significant due to the relatively small sample size. Another single institution study reports an increase in median survival time in 16 patients with NFpNET and distant metastases undergoing resection of their primary tumor ( 3 years, CI 2.2-8.0) versus 80 patients who did not undergo surgery (1.8 years, CI 1.4-2.7) but this finding was not statistically significant, possibly due to the relatively small sample size (14). Additionally, this study did not mention tumor grading and could therefore not stratify survival according to this factor.

Younger age and lower tumor grade have consistently been found to be associated with longer survival time in patients with pNET $(2,9,13-16)$. It remains unclear if the survival advantage in younger patients is due to better physiologic reserves and ability to sustain more aggressive treatment, clinician bias in treating older patients with less aggressive therapy regimens or more indolent tumor biology in younger patients. Patients with grade III pNET have a poor overall survival and surgical therapy for metastastic disease is generally not recommended in this patient group (7). Another significant factor found to be associated with longer survival time on multivariable analyses was the location of the primary tumor in the body/tail of the pancreas. Different tumor biology, separate lymphatic drainage basins, or less likelihood of developing biliary and pancreatic and visceral vessel compromise when compared to pancreatic head tumors, may explain this finding. This hypothesis is also supported by the fact that patients who didn't undergo surgery and had their primary tumor in the body/tail lived longer than those who had their tumors in the head of the pancreas. Year of diagnosis during or after 2003 was also shown to be an independent variable associated with prolonged survival. We used specific time points (2003 and 2008) for our analysis in order to do a comparison of early vs. late diagnosis year since the data ranged over decades. In particular, the year 2003 was chosen since patients diagnosed with NFpNET after 2003 were coded with the AJCC staging in SEER. It is not entirely surprising that patients diagnosed during or after 2003 fared better than those diagnosed at a previous time point. This is less likely due to a change in biological behavior of the tumors, but rather to clinicians' detection, understanding and classification of pNET as well as the development of better imaging and targeted therapies, potentially leading to better treatment adjustments and more aggressive treatment strategies, ultimately leading to prolonged survival in those patients diagnosed after 2003. 
Although, the observed longer survival time in patients after surgical removal of primary tumor may have been due to the fact that patient who had an operation could have received additional life-prolonging therapy, especially in more recent years, the exact reasons are unknown and will need to be studied further in order to prove the efficacy of surgical treatment in patients with NFpNET and distant metastases.

There are several limitations to our study. The retrospective analysis is subject to a selection bias as we had no information on why specific treatment strategies were used. Moreover, the classification of functional versus non-functional tumors may not be accurate. SEER does not provide information on a uniformly applied pathological grading system and miscoding of grading could have occurred. For instance, 39\% of NFpNET were graded as grade III, which is higher than the reported number of $22 \%$ in a study from the National Cancer Database including 9821 patients with NET (17). SEER also does not give information on the use of adjuvant therapies for residual disease commonly applied in neuroendocrine tumors. This limits our ability to perform a meaningful analysis regarding post-procedural therapies that may have influenced survival in either group. However, one could assume that the impact of adjuvant therapies on survival reported in SEER is low, since most effective therapies such as transarterial (chemo-) embolisation, somatostatin- or radiolabeled somatostatin analogues as well as targeted therapy are newer treatment modalities that have only recently been used (18). Lastly, we do not know the precise location and number of distant metastases to control for this confounding variable, although previous data suggests that a vast majority of distant metastases in NFpNET occur in the liver $(14,17)$.

Despite these limitations we believe that clinicians should give consideration to the removal of primary tumor in well- to moderately differentiated NFpNET with distant metastases, especially in younger patients and if the tumor is localized in the body or tail of the pancreas as this surgical procedure is associated with lower morbidity and mortality (19).

\section{CONCLUSIONS}

This study suggests that surgical removal of primary NFpNET is associated with prolonged survival in patients with distant metastases. This treatment option should however be evaluated on an individual base for each patient and could be incorporated with other treatment strategies according to the clinician's judgment. Future prospective trials are needed to confirm these findings.

\section{References}

1. Oberg K. Pancreatic endocrine tumors. Seminars in oncology. 2010 Dec; 37(6):594-618. [PubMed: 21167379]

2. Franko J, Feng W, Yip L, Genovese E, Moser AJ. Non-functional neuroendocrine carcinoma of the pancreas: incidence, tumor biology, and outcomes in 2,158 patients. Journal of gastrointestinal surgery : official journal of the Society for Surgery of the Alimentary Tract. 2010 Mar; 14(3):5418. [PubMed: 19997980]

3. Yao JC, Eisner MP, Leary C, Dagohoy C, Phan A, Rashid A, et al. Population-based study of islet cell carcinoma. Annals of surgical oncology. 2007 Dec; 14(12):3492-500. [PubMed: 17896148] 
4. Kulke MH, Anthony LB, Bushnell DL, de Herder WW, Goldsmith SJ, Klimstra DS, et al. NANETS treatment guidelines: well-differentiated neuroendocrine tumors of the stomach and pancreas. Pancreas. 2010 Aug; 39(6):735-52. [PubMed: 20664472]

5. Krampitz GW, Norton JA. Pancreatic neuroendocrine tumors. Current problems in surgery. 2013 Nov; 50(11):509-45. [PubMed: 24206780]

6. Niina Y, Fujimori N, Nakamura T, Igarashi H, Oono T, Nakamura K, et al. The current strategy for managing pancreatic neuroendocrine tumors in multiple endocrine neoplasia type 1 . Gut and liver. 2012 Jul; 6(3):287-94. [PubMed: 22844555]

7. Pavel M, Baudin E, Couvelard A, Krenning E, Oberg K, Steinmuller T, et al. ENETS Consensus Guidelines for the management of patients with liver and other distant metastases from neuroendocrine neoplasms of foregut, midgut, hindgut, and unknown primary. Neuroendocrinology. 2012; 95(2):157-76. [PubMed: 22262022]

8. Rindi, GAR.; Bosman, FT. Nomenclature and classification of neuroendocrine neoplasms of the digestive system. Bodman, FTCF.; Hruban, RH., editors. Lyon: International Agency for Research on Cancer (IRAC); 2010. p. 10-145.

9. Ito H, Abramson M, Ito K, Swanson E, Cho N, Ruan DT, et al. Surgery and staging of pancreatic neuroendocrine tumors: a 14-year experience. Journal of gastrointestinal surgery : official journal of the Society for Surgery of the Alimentary Tract. 2010 May; 14(5):891-8. [PubMed: 20224984]

10. National Cancer Institute, U.S. National Institutes of Health. [accessed May 21, 2012] Surveillance Epidemiology and End Results: ICD-O-3 Coding Materials. 2012. Available at 2012. Available from: http://seer.cancer.gov/icd-o-3/

11. Office of Management and Budget. [accessed May 21, 2012] Revisions to the Standards for the Classification of Federal Data on Race and Ethnicity. 2003. Available at Washington, DC2003. Available from: http://www.whitehouse.gov/omb/fedreg_1997standards/

12. Rindi G, Kloppel G, Alhman H, Caplin M, Couvelard A, de Herder WW, et al. TNM staging of foregut (neuro)endocrine tumors: a consensus proposal including a grading system. Virchows Archiv : an international journal of pathology. 2006 Oct; 449(4):395-401. [PubMed: 16967267]

13. Bettini R, Mantovani W, Boninsegna L, Crippa S, Capelli P, Bassi C, et al. Primary tumour resection in metastatic nonfunctioning pancreatic endocrine carcinomas. Digestive and liver disease : official journal of the Italian Society of Gastroenterology and the Italian Association for the Study of the Liver. 2009 Jan; 41(1):49-55.

14. Solorzano CC, Lee JE, Pisters PW, Vauthey JN, Ayers GD, Jean ME, et al. Nonfunctioning islet cell carcinoma of the pancreas: survival results in a contemporary series of 163 patients. Surgery. 2001 Dec; 130(6):1078-85. [PubMed: 11742342]

15. Curran T, Pockaj BA, Gray RJ, Halfdanarson TR, Wasif N. Importance of lymph node involvement in pancreatic neuroendocrine tumors: impact on survival and implications for surgical resection. Journal of gastrointestinal surgery : official journal of the Society for Surgery of the Alimentary Tract. 2015 Jan; 19(1):152-60. [PubMed: 25118642]

16. Hill JS, McPhee JT, McDade TP, Zhou Z, Sullivan ME, Whalen GF, et al. Pancreatic neuroendocrine tumors: the impact of surgical resection on survival. Cancer. 2009 Feb 15; 115(4): 741-51. [PubMed: 19130464]

17. Bilimoria KY, Tomlinson JS, Merkow RP, Stewart AK, Ko CY, Talamonti MS, et al. Clinicopathologic features and treatment trends of pancreatic neuroendocrine tumors: analysis of 9,821 patients. Journal of gastrointestinal surgery : official journal of the Society for Surgery of the Alimentary Tract. 2007 Nov; 11(11):1460-7. discussion 7-9. [PubMed: 17846854]

18. Rossi RE, Massironi S, Conte D, Peracchi M. Therapy for metastatic pancreatic neuroendocrine tumors. Annals of translational medicine. 2014 Jan.2(1):8. [PubMed: 25332984]

19. McPhee JT, Hill JS, Whalen GF, Zayaruzny M, Litwin DE, Sullivan ME, et al. Perioperative mortality for pancreatectomy: a national perspective. Annals of surgery. 2007 Aug; 246(2):24653. [PubMed: 17667503] 
A

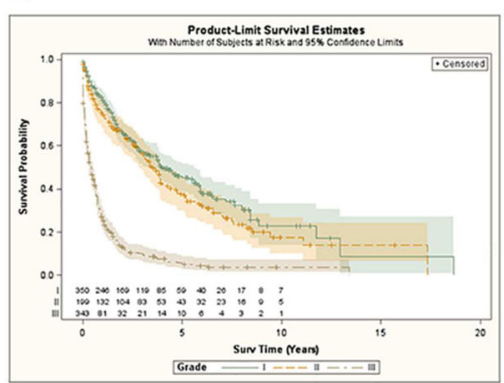

D

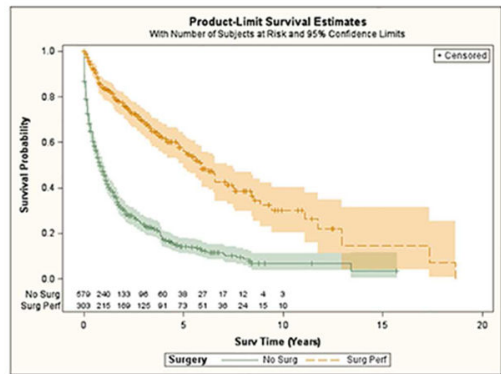

B

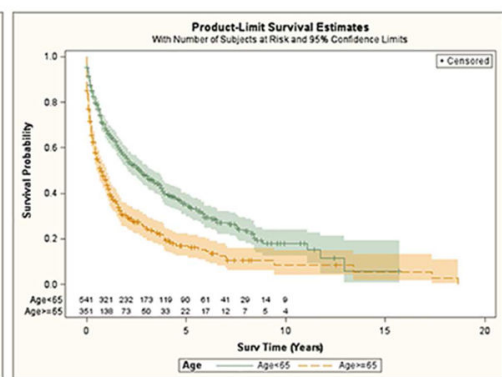

E

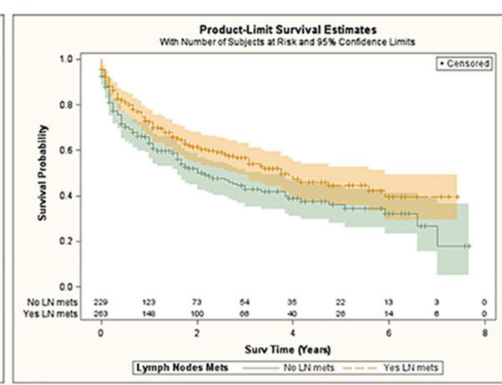

C

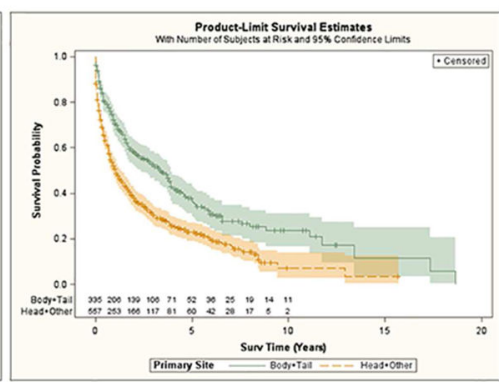

F

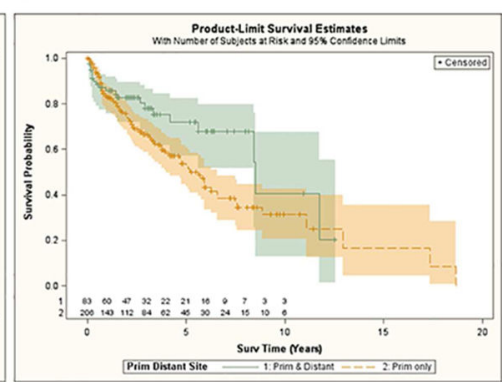

Figure 1.

Kaplan-Meier curves according to grade $(\mathrm{p}<0.0001)(\mathbf{A})$, age $(\mathrm{p}<0.0001)(\mathbf{B})$, tumor location $(\mathrm{p}<0.0001)(\mathbf{C})$, lymph node metastases $(\mathrm{p}=0.013)(\mathbf{D})$, surgery $(\mathrm{p}<0.0001)(\mathbf{E})$ and resection site $(\mathrm{p}=0.052)(\mathbf{F})$ (Rows of values above the $\mathrm{X}$ axes indicate the number of patients at risk at selected time points; shaded areas represent $95 \%$ confidence intervals). 


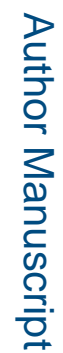

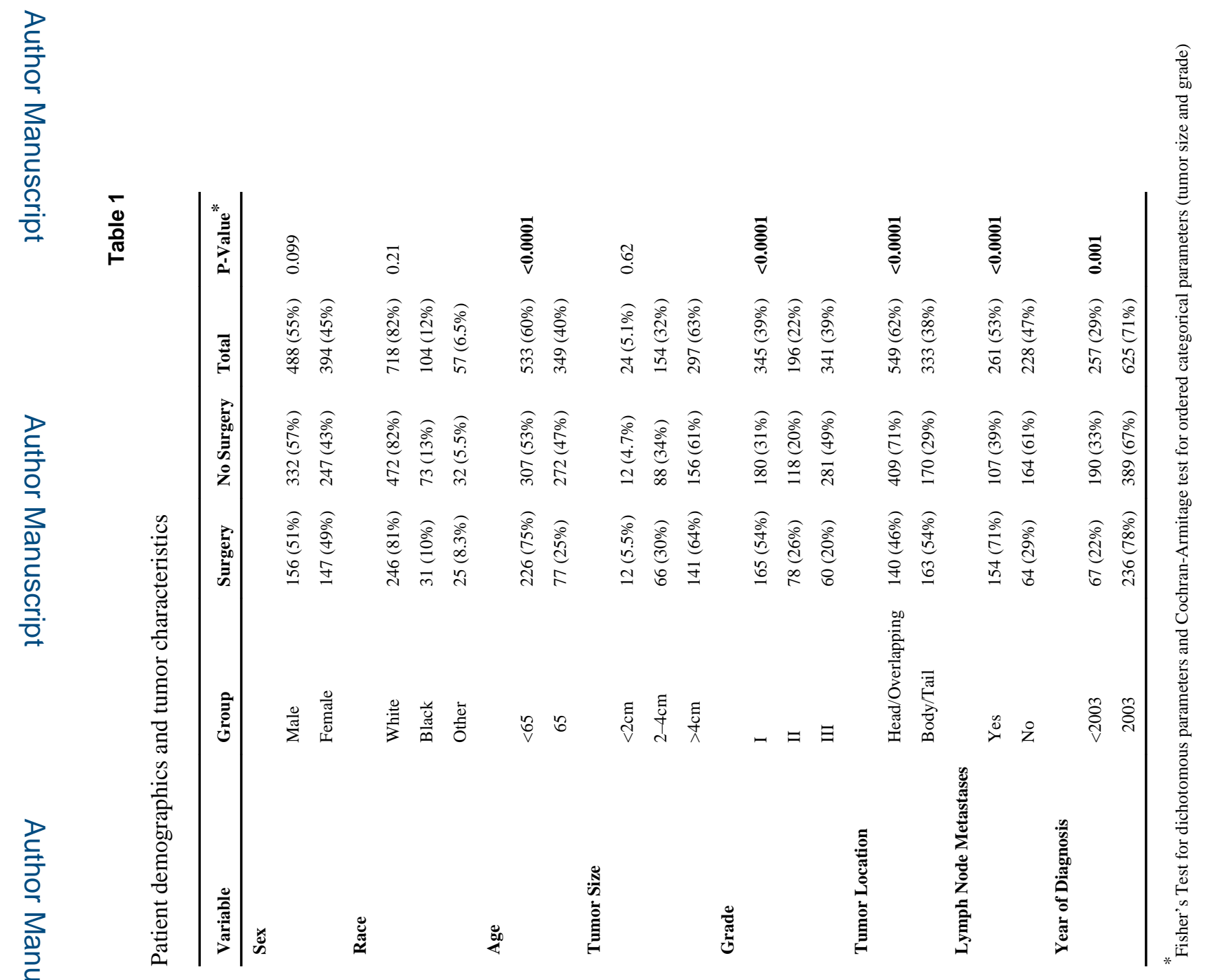


Table 2

Association of clinical and pathological variables with survival

\begin{tabular}{|c|c|c|c|c|}
\hline Variable & Groups & $\mathbf{N}$ & Median & $95 \%$ CI \\
\hline \multicolumn{5}{|l|}{ Grade } \\
\hline & I & 350 & 4.00 & $3.33-5.58$ \\
\hline & II & 199 & 3.50 & $2.83-4.00$ \\
\hline & $\mathrm{I} / \mathrm{II}$ & 549 & 3.83 & $3.33-4.50$ \\
\hline & III & 343 & 0.42 & $0.33-0.50$ \\
\hline \multicolumn{5}{|l|}{ Age } \\
\hline & $<50$ & 208 & 3.08 & $2.17-3.83$ \\
\hline & $50-64$ & 333 & 2.50 & $1.50-3.50$ \\
\hline & $<65$ & 541 & 2.67 & $2.08-3.42$ \\
\hline & $\lcm{65}$ & 351 & 0.75 & $0.50-1.00$ \\
\hline \multicolumn{5}{|l|}{ Race } \\
\hline & Black & 105 & 1.50 & $0.67-2.50$ \\
\hline & White & 727 & 1.67 & $1.33-1.92$ \\
\hline & Other & 57 & 2.00 & $1.08-3.92$ \\
\hline \multicolumn{5}{|l|}{ Sex } \\
\hline & Female & 398 & 1.92 & $1.42-2.42$ \\
\hline & Male & 494 & 1.50 & $1.67-1.75$ \\
\hline \multicolumn{5}{|c|}{ Year of Diagnosis } \\
\hline & $<2003$ & 263 & 0.92 & $0.75-1.17$ \\
\hline & 2003-2008 & 275 & 1.83 & $1.33-2.83$ \\
\hline & $\geq 2008$ & 355 & 2.58 & 1.75-UD * \\
\hline & 2003 & 629 & 2.25 & $1.75-3.00$ \\
\hline \multicolumn{5}{|c|}{ Tumor Location } \\
\hline & Head & 279 & 1.00 & $0.67-1.42$ \\
\hline & Overlapping & 278 & 1.67 & $0.83-1.50$ \\
\hline & Head/Overlapping & 557 & 1.08 & $0.83-1.33$ \\
\hline & Body & 92 & 3.42 & $1.67-4.50$ \\
\hline & Tail & 243 & 3.50 & $2.08-4.00$ \\
\hline & Body/Tail & 335 & 3.42 & $2.33-3.92$ \\
\hline \multicolumn{5}{|c|}{ Tumor Size (cm) } \\
\hline & $<2$ & 24 & 2.33 & $0.33-\mathrm{UD}^{*}$ \\
\hline & $2-3.99$ & 156 & 3.33 & $1.75-5.92$ \\
\hline & $\geq 4$ & 298 & 3.08 & $2.00-3.92$ \\
\hline \multicolumn{5}{|c|}{ Lymph Node Metastases } \\
\hline & Yes & 263 & 3.75 & $2.83-5.92$ \\
\hline & No & 229 & 2.17 & $1.58-3.33$ \\
\hline \multicolumn{5}{|l|}{ Surgery } \\
\hline & Yes & 303 & 5.42 & $5.00-7.17$ \\
\hline & Not Recommended & 500 & 0.92 & $0.67-1.08$ \\
\hline
\end{tabular}

Surgery. Author manuscript; available in PMC 2017 January 01. 


\begin{tabular}{lllll}
\hline Variable & Groups & N & Median & 95\% CI \\
\hline \multirow{3}{*}{ Site Specific Surgery } & Recommended but not performed & 79 & 0.58 & $0.25-0.83$ \\
& No & 579 & 0.83 & $0.67-1.00$ \\
& Local, enucleation & & & \\
& Pancreatectomy, not otherwise specified & 30 & 3.25 & $1.00-8.83$ \\
& Partial Pancreatectomy & 221 & 6.58 & $4.75-8.42$ \\
& Total Pancreatectomy & 28 & 17.30 & $3.42-17.3$ \\
& & & & \\
& & 83 & 8.50 & $8.42-$ UD \\
& Primary Site Surgery & 206 & 5.58 & $4.00-6.58$ \\
\hline
\end{tabular}

*UD: undefined 

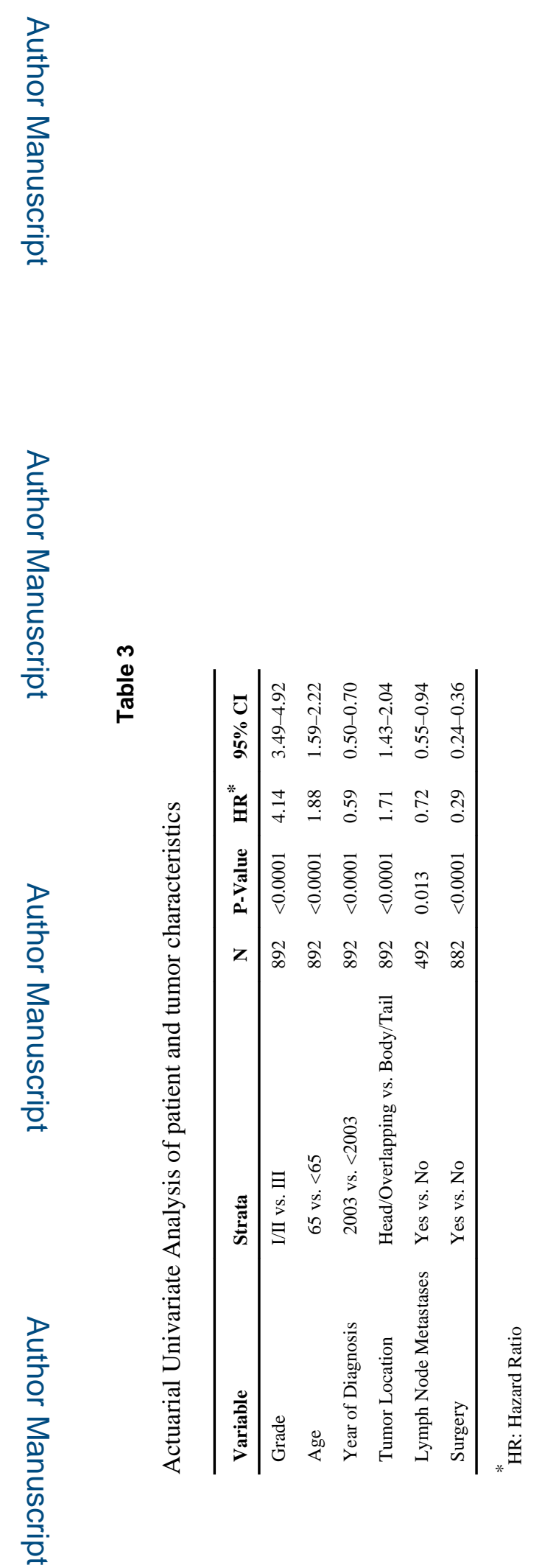

Surgery. Author manuscript; available in PMC 2017 January 01. 
Table 4

Multivariable analysis of patient and tumor characteristics ( $\mathrm{n}=882$ )

\begin{tabular}{lccc}
\hline Variable & P-Value & HR $^{*}$ & $\mathbf{9 5 \%}$ CI \\
\hline Grade I/II & $<0.0001$ & 0.31 & $0.25-0.37$ \\
Age $<65$ & $<0.0001$ & 0.67 & $0.57-0.80$ \\
Year of Diagnosis 2003 & 0.0003 & 0.73 & $0.62-0.87$ \\
Tumor location: Body/Tail & 0.0095 & 0.78 & $0.65-0.94$ \\
Surgery & $<0.0001$ & 0.41 & $0.33-0.50$ \\
\hline
\end{tabular}

* HR: Hazard Ratio 
Table 5

Cox model results for overall survival according to surgery

\begin{tabular}{lllll}
\hline Group & Variable & P-Value & HR $^{*}$ & $\mathbf{9 5 \%} \mathbf{C I}$ \\
\hline Surgery $(\mathrm{n}=303)$ & Year of Diagnosis & 0.015 & 0.62 & $0.42-0.91$ \\
& Grade & $<0.0001$ & 0.24 & $0.16-0.35$ \\
No Surgery $(\mathrm{n}=579)$ & Year of Diagnosis & 0.008 & 0.77 & $0.64-0.93$ \\
& Age & $<0.0001$ & 0.66 & $0.54-0.79$ \\
& Tumor location: Body/Tail & 0.050 & .81 & $0.65-1.00$ \\
& Grade & $<0.0001$ & 0.33 & $0.27-0.40$ \\
\hline
\end{tabular}

*HR: Hazard Ratio 
Table 6

Logistic regression results of surgery stratified by year of diagnosis

\begin{tabular}{lllll}
\hline YOD & Variable & P-Value & OR $^{*}$ & 95\% CI \\
\hline$<2003(\mathrm{n}=257)$ & Grade & 0.0003 & 3.37 & $1.75-6.47$ \\
& Age & 0.070 & 1.87 & $0.95-3.68$ \\
& Tumor location: Body/Tail & 0.0003 & 3.17 & $1.71-5.91$ \\
\multirow{2}{*}{$003(\mathrm{n}=625)$} & Grade & $<0.0001$ & 2.93 & $1.96-4.36$ \\
& Age & $<0.0001$ & 2.29 & $1.58-3.30$ \\
& Tumor location: Body/Tail & $<0.0001$ & 2.12 & $1.50-3.01$ \\
\hline
\end{tabular}

* Odds Ratio 